Communication

\title{
Additive-Free Baeyer-Villiger Oxidation of Cyclic Ketone Catalyzed by Carboxylic-Functionalized Poly(Ionic Liquids) and Polyoxometalate Ionic Self-Assemblies
}

\author{
Xinzhong Li ${ }^{1,2,3,4, *}$, Hanyu Xue ${ }^{1,2,3}$, Qi Lin ${ }^{1,2,3}$ and Aimin $\mathrm{Yu}^{4}$ \\ 1 Department of Materials Engineering, Ocean College, Minjiang University, Fuzhou 350108, China; \\ Hanyu@mju.edu.cn (H.X.); Qilin@mju.edu.cn (Q.L.) \\ 2 Fujian Engineering and Research Centre of New Chinese Lacquer Material, Ocean College, \\ Minjiang University, Fuzhou 350108, China \\ 3 Fujian Provincal University Engineering Research Centre of Green Materials and Chemical Engineering, \\ Ocean College, Minjiang University, Fuzhou 350108, China \\ 4 Faculty of Science, Engineering and Technology, Swinburne University of Technology, \\ Hawthorn, VIC 3122, Australia; aiminyu@swin.edu.au \\ * Correspondence: lixinzhong77@126.com
}

Received: 10 December 2019; Accepted: 11 January 2020; Published: 16 January 2020

check for updates

\begin{abstract}
Two carboxylic functionalized poly(ionic liquids) and polyoxometalate ionic self-assemblies with tunable amphiphilic and oxidative activity and porous structure were designed and synthesized. They were used as efficient heterogeneous catalysts exhibiting excellent catalytic activity and quite stable reusability in additive-free Baeyer-Villiger oxidation of cyclic ketones, directly using $30 \% \mathrm{H}_{2} \mathrm{O}_{2}$ solution in liquids-phase. Under optimum conditions, the corresponding lactones were obtained with yields ranging from 59.6 to $90.5 \%$. The unique combination of carboxylic group with polyoxometalate anion, amphiphilic property, and porous structure is revealed to be responsible for their excellent catalytic performances in oxidation.
\end{abstract}

Keywords: poly(ionic liquids); polyoxomatalate; cyclic ketone; Baeyer-Villiger oxidation; lactone

\section{Introduction}

Recently, the growing concerns about plastic's environmental footprint and its manufacture from bio-renewable feedstocks have received considerable attention from industry and academic communities [1,2]. One of the most rapidly developing fields is aliphatic polyester with excellent hydrolytic degradability [3,4] like polylactones and polylactides, which have been widely applied in biomedicine and engineering fields. Since the efficient synthetic pathway for the preparation of aliphatic polyester is ring-opening polymerization of lactone monomer, Baeyer-Villiger (BV) oxidation represents a powerful synthetic tool for the construction of lactone from the corresponding ketone. It is carried out in no catalytic ways using organic peracids, such as $m$-chloroperbenzoic [5] and perdecanoic acid [6] in more than stoichiometric amounts in complex solutions, which are shock sensitive, explosive, with inadvertently low reactivity and selectivity in oxidation, and which generate equimolar carboxylic acid as waste. Therefore, there is a compelling demand for the development of more sustainable and practical BV methods using molecular oxygen or $\mathrm{H}_{2} \mathrm{O}_{2}$ as green oxidant. Due to the lower oxidation potential of $\mathrm{H}_{2} \mathrm{O}_{2}$, the catalyst is usually required to promote oxidation via carbonyl or $\mathrm{H}_{2} \mathrm{O}_{2}$ activation. For example, heterogeneous catalysts based on Lewis acidic transition metal (like Sn [7,8], Fe [9], Mg [10], Mn [11]), sold acid [12], sulfonic acid functionalized silica nanoparticle [13], 
carboxylic multi-walled carbon nanotube [14], and macroporous CNT-sieve-composite-supported polyoxometalate [15] have been reported; however, these methods are confronted with the drawbacks of low activity or selectivity and complicated preparation processes, using high concentrations of $\mathrm{H}_{2} \mathrm{O}_{2}$ and so on.

Polymeric ionic liquids (PILs), as a kind of designable multifunctional polyelectrolyte, combine the properties of polymer and ionic liquid. By changing the anion and cation, a variety of functional materials with unique physicochemical properties and catalytic activities have been fabricated [16]. For example, introducing various polyoxometalate (POM) anions to provide a series of PILs-based POM composites or hybrids, these compounds acted as green heterogeneous catalysts with high catalytic activity and selectivity in olefins epoxidation with $\mathrm{H}_{2} \mathrm{O}_{2}$ [17], electrocatalytic water oxidation [18], and sulfides selective oxidation [19], starting from $\mathrm{SO}_{3} \mathrm{H}$ functionalized acidic ionic liquids monomer via controllable polymerization, to prepare microporous, mesoporous, microporous, and hierarchically nanoporous PILs with strong acid strength and use them as efficient solid acids in various acid-catalyzed reactions [20-23]. Nevertheless, the self-assembled POM and PILs composites or hybrids are always nonporous. The hydrophilic or hydrophobic frameworks of polymeric cation usually hinder the interaction of the POM catalytic center with the hydrophobic substrate and limit the heterogeneous catalytic activity in mass-transfer control.

Aiming to develop a green and sustainable protocol for BV oxidation with $\mathrm{H}_{2} \mathrm{O}_{2}$, in this context, two novel multifunctional PILs and POM ionic self-assemblies (denoted as COOH-PIL@P-POM and COOH-PIL@Si-POM) (Scheme 1) with tunable amphiphilic and oxidative properties were fabricated by ionic self-assembling of carboxylic functionalized PILs with Keggin type $\mathrm{H}_{3} \mathrm{PW}_{12} \mathrm{O}_{40}$ and $\mathrm{H}_{3} \mathrm{PW}_{12} \mathrm{O}_{40}$ in water. The as-synthesized ionic self-assemblies served as efficient heterogeneous catalysts exhibiting excellent catalytic activity and stable reusability in additive-free $\mathrm{BV}$ oxidation of cyclic ketones in the presence of $30 \% \mathrm{H}_{2} \mathrm{O}_{2}$ under liquids-phase. The corresponding lactones were obtained with yields ranging from $59.6 \%$ to $90.5 \%$. Two ionic self-assemblies can be reused for five times after simple work-up and without noticeable decrease of activity.

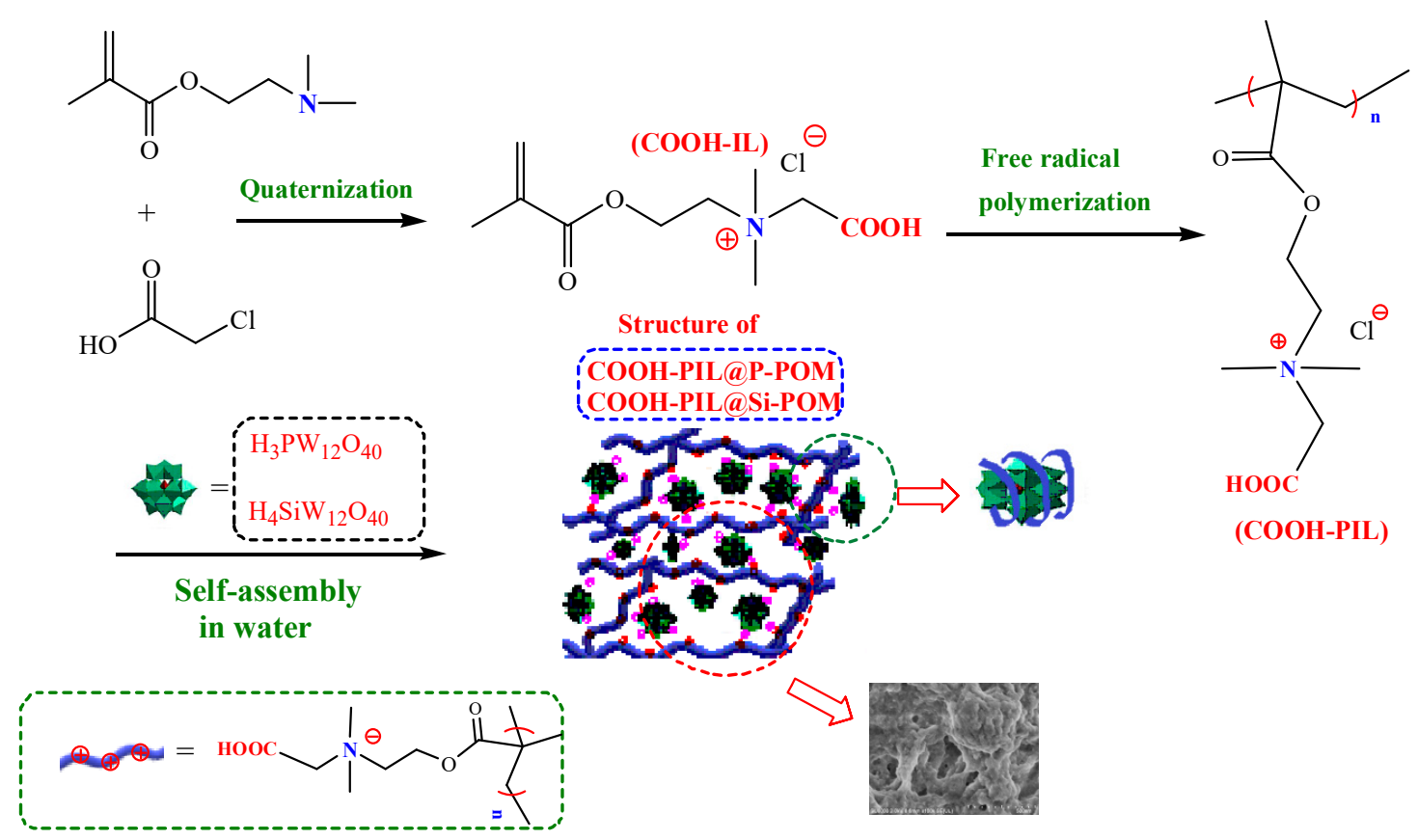

Scheme 1. Additive-free BV oxidation of cyclic ketones with $\mathrm{H}_{2} \mathrm{O}_{2}$ catalyzed by carboxylic. functionalized PILs and POM ionic self-assemblies COOH-PIL@P-POM and COOH-PIL@Si-POM. 


\section{Results and Discussion}

\subsection{Synthesis and Characterization of Two Ionic Self-Assemblies}

COOH-PIL@P-POM and COOH-PIL@Si-POM were synthesized using 2-(dimethylamino)ethyl methyacrylate, chloroacetic acid, $\mathrm{H}_{3} \mathrm{PW}_{12} \mathrm{O}_{40}$ and $\mathrm{H}_{4} \mathrm{SiW}_{12} \mathrm{O}_{40}$ as starting materials via quaternization, free radical polymerization, and ionic self-assembly in water. 2-(dimethylamino)ethyl methyacrylate as a kind of industrial available chemical shows strong basicity; this nature causes the quaternization to be carried out easily and to proceed completely under mild conditions. Importantly, the resulting ionic liquid monomer not only can be easily polymerized but also produce a polymer with linear long chain quaternary ammonium structure. The reason for choosing $\mathrm{H}_{3} \mathrm{PW}_{12} \mathrm{O}_{40}, \mathrm{H}_{4} \mathrm{SiW}_{12} \mathrm{O}_{40}$ as the suitable counter POM lies in: (1) two POM exhibited good performance in catalytic activity in BV oxidation via generate peroxo intermediate in situ with $\mathrm{H}_{2} \mathrm{O}_{2}$, and their oxidative activity could be regulated by the acidic content and acidity [15] and (2) two POM anions have the size of $1 \mathrm{~nm} \times 1 \mathrm{~nm}$, together with their high negative charge density, which is beneficial for the adequate coating of PILs on their surfaces and the formation of an excellent amphiphilic microenvironment. On the other hand, two POM anions could function as pillar type nodes or crosslinking agents embedded and supported in the 2D net-like structure formed by the linear long chain polymeric quaternary ammonium cation and thereby create an open 3D network structure with hierarchical porosity. In addition, the synthesized carboxylic functionalized PILs and two POMs both have excellent solubility in water, but the resulting ionic self-assemblies are insoluble. Therefore, the self-assembly in water ensures a product with high purity. The produced two carboxylic functionalized ionic self-assemblies show oxidative, acidic, and excellent amphiphilic activity at the same time, and these properties can be well tuned by the polymerization degree and the number and type of POM anion.

Figure 1a shows the XRD patterns of two ionic self-assemblies; these samples exhibit two broad peaks centered at $5^{\circ}$ to $10^{\circ}$ and $20^{\circ}$ to $40^{\circ}$, which could be attributed to the characteristic peaks of Keggin type POM, suggesting the crystal structure and Keggin configuration of POM anion is still well retained. As for the facts of the noticeable decrease in the intensities and no peaks appearing in the high angel areas, they could be ascribed to the POM anions being well dispersed in the polycationic frameworks via rearrangement during the self-assembly.

(a)

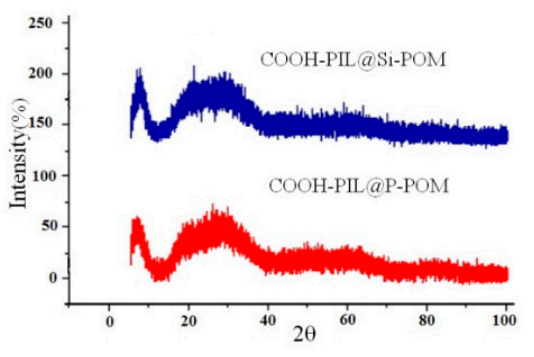

(b)
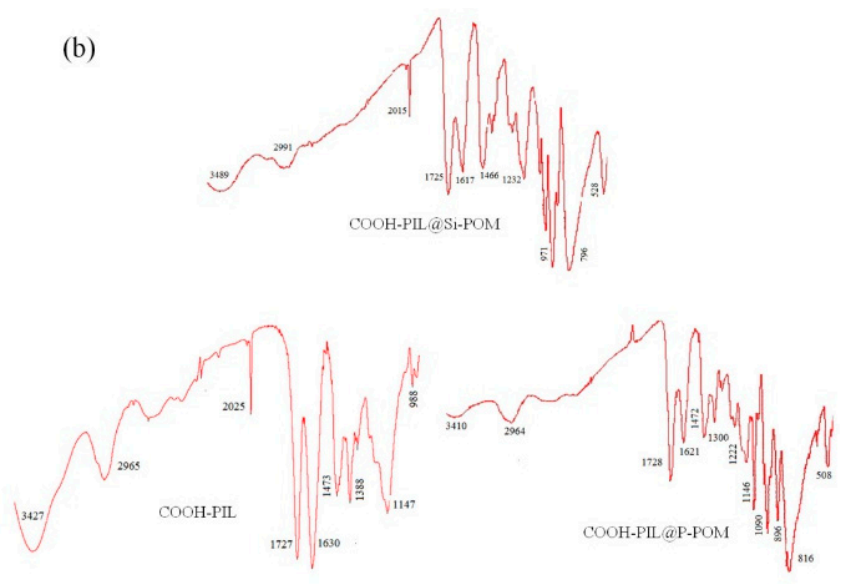

Figure 1. (a) XRD patterns of COOH-PIL@P-POM and COOH-PIL@Si-POM; (b) FT-IR spectra of COOH-PIL@P-POM, COOH-PIL@Si-POM, and COOH-PIL.

Figure $1 \mathrm{~b}$ shows the infrared data of two ionic self-assemblies and carboxylic functionalized PIL. The absorption bonds appeared at 1725, 1630,1470, and $1140 \mathrm{~cm}^{-1}$, which could be assigned to the vibrations of $\mathrm{C}=\mathrm{O}$ groups of ester and carboxylic moiety, $\mathrm{C}-\mathrm{N}$ stretching, and $\mathrm{C}-\mathrm{O}$ vibration, respectively, suggesting the polycationic frameworks was introduced successfully. In the wavenumber regions of $750-1200 \mathrm{~cm}^{-1}, \mathrm{PW}_{12} \mathrm{O}_{40}{ }^{-3}$ gave four characteristic bands at $1080,978,896$, and $816 \mathrm{~cm}^{-1} . \mathrm{SiW}_{12} \mathrm{O}_{40}{ }^{-4}$ 
gave five characteristic bands at 1015, 972, 920, 882, and $796 \mathrm{~cm}^{-1}$. These facts combined with XRD results prove that the basic structure and geometry of the Keggin type POM anion are well-preserved. The vibration bands of POM anion in two ionic self-assemblies are slightly shifted in comparison with free POM, which result from the strong ionic interaction between the polymeric quaternary ammonium cation and POM anion. In addition, compared with the fresh ionic self-assemblies, there is no notable difference shown in the FT-IR spectrum of the used ionic self-assemblies after five recycles, indicating the highly chemical and structural stability of two ionic self-assemblies.

TG curves of COOH-PIL@P-POM and COOH-PIL@Si-POM are presented in Figure 2. All samples show similar changing trend from $30{ }^{\circ} \mathrm{C}$ to $400{ }^{\circ} \mathrm{C}$. There is an initial weight loss of about $1 \%$ to $1.5 \%$ that can be observed up to $100^{\circ} \mathrm{C}$, which is attributed to the adsorbed moisture and hydrated water of POM anions and is almost stable up to $250{ }^{\circ} \mathrm{C}$ (the loss of weigh less than $5 \%$ ). The main weight loss occurred around $250{ }^{\circ} \mathrm{C}$, which indicates the slow decomposition of the counter polymeric organic cation.

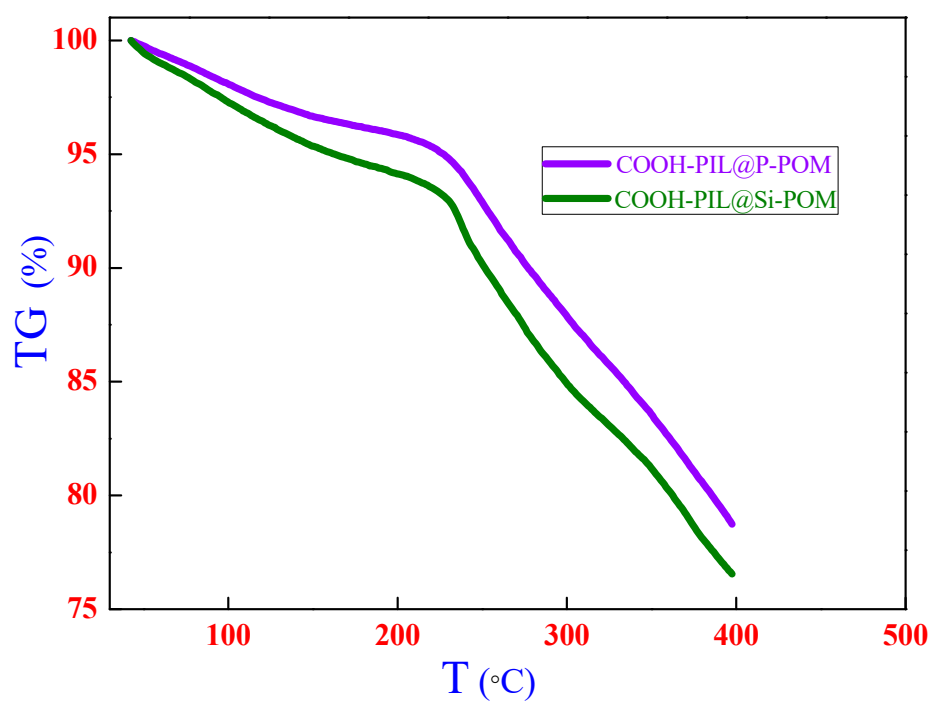

Figure 2. TG patterns of COOH-PIL@P-POM and COOH-PIL@Si-POM. The viscosity average FW of 23,000 .

SEM images of two self-assemblies with different viscosity average molecular weight were shown in Figure 3a-d. As it can be seen, the prepared samples displayed the aggregation morphology of small spherical and block nanoparticles with a size of about hundreds of nanometers. These nanoparticles further packed and linked each other to form irregular secondary particles with the size of a micrometer. It is obvious that abundant net-like hierarchical mesopores and macropores can be found in the secondary particles. All samples have waxy smooth surface without observable pores, indicating the coating of polymeric quaternary ammonium cation around the surface of POM anion is sufficient and uniform, and POM anions are completely entrapped. The effect of polymeric quaternary ammonium cation on the morphology of ionic self-assembly is remarkable. With an increase in polymerization degree, the size of particle improved, due to the agglomeration of polymeric cation on the surface of POMs anion. When the POM anion changed from $\mathrm{PW}_{12} \mathrm{O}_{40}{ }^{-3}$ to $\mathrm{SiW}_{12} \mathrm{O}_{40}{ }^{-4}$, there was a remarkable improvement in degree of cross-linking between particles, which could be ascribed to the increase of negative charge of POM anion. In addition, no separate aggregation of PILs was found, indicating that the PILs coating has occurred only on the surface of POM anion.

Figure 4 shows the $\mathrm{N}_{2}$ adsorption-desorption isotherms and the corresponding pore size distribution curves of COOH-PIL@P-POM. According to Brunauer-Deming-Deming-Teller classification, these isotherms can be categorized as type IV, indicating the presence of mesopores in ionic self-assembly. Pore diameter curve manifests wide pore size distribution from $5 \mathrm{~nm}$ to $120 \mathrm{~nm}$ and a broad peak centered at ca. $30-60 \mathrm{~nm}$. These pores located at the empty space inside the 
hierarchical architectures, which is consistent with the results of SEM. The BET specific surface area of COOH-PIL@P-POM is $26.46 \mathrm{~m}^{3} / \mathrm{g}$. The high specific surface area and hierarchical pore structure are favorable for COOH-PIL@P-POM used as effective heterogeneous catalysis in BV oxidation.

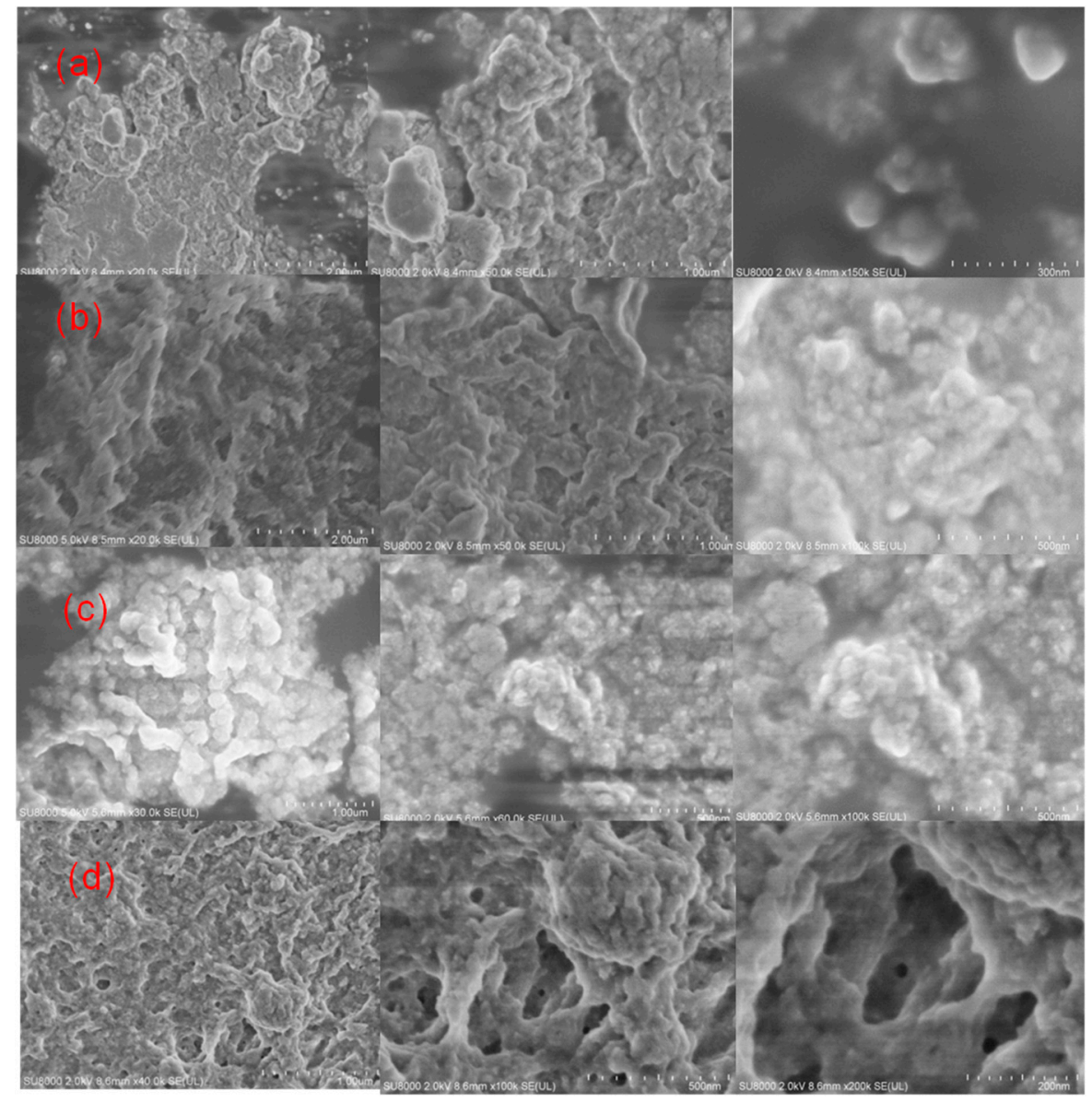

Figure 3. SEM images of two ionic self-assemblies. (a) COOH-PL@P-POM, the viscosity average FW of the counter polycation is 23,000; (b) COOH-PL@Si-POM, the viscosity average FW of the counter polycation is 23,000; (c) COOH-PL@P-POM, the viscosity average FW of the counter polycation is 15,000; (d) COOH-PL@Si-POM, the viscosity average FW of the counter polycation is 15,000.
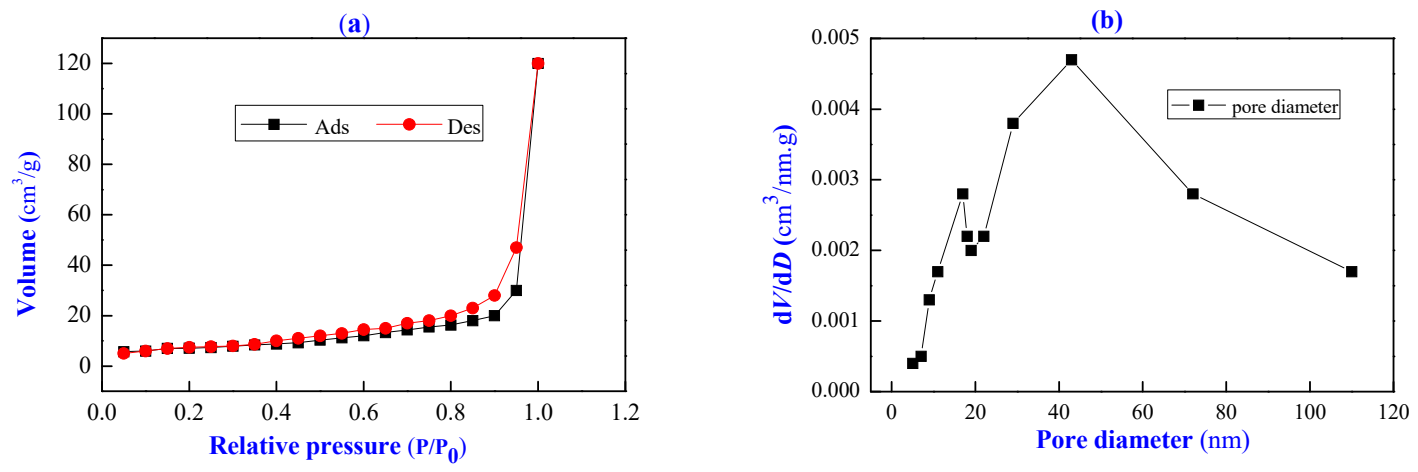

Figure 4. $\mathrm{N}_{2}$ adsorption/desorption isotherms (a) and pore size distribution (b) curves of COOH-PIL@P-POM (FW = 23,000).

\subsection{Catalytic Performance in Additive-Free BV Oxidation}

Since $\varepsilon$-caprolactone is the most important lactone used in industry with global consumption of over $30 \mathrm{k}$ ton annually, initially, the oxidation of cyclohexanone under atmospheric pressure catalyzed 
by two ionic self-assemblies was evaluated as model reaction. Various reaction parameters including the amount of catalyst, temperature, the molar ratio of $\mathrm{H}_{2} \mathrm{O}_{2}$ to ketone, time, and solvent were investigated. Under optimum conditions, these results were listed in Table 1. As shown in Table 1 (entries 5-8), both ionic self-assemblies exhibited notable advantages of lower usage of catalysts, high conversion and selectivity, shorter reaction time, and lower reaction temperature.

Table 1. The catalytic performance of various catalysts in Baeyer-Villiger (BV) oxidation of cyclohexanone with $\mathrm{H}_{2} \mathrm{O}_{2}$.

\begin{tabular}{|c|c|c|c|c|c|}
\hline Entry & Catalyst & Reaction Conditions & Conversion (\%) & Selectivity (\%) & Yield (\%) \\
\hline 1 & blank & $\begin{array}{c}5 \text { mmol ketone, } \mathrm{H}_{2} \mathrm{O}_{2} 15 \mathrm{mmol}, \\
\text { DCE } 15 \mathrm{~mL}, 60^{\circ} \mathrm{C}, 4 \mathrm{~h}\end{array}$ & - & - & - \\
\hline 2 & $\mathrm{H}_{3} \mathrm{PW}_{12} \mathrm{O}_{40}$ & $\begin{array}{l}5 \mathrm{mmol} \text { ketone, catalyst } \\
0.0025 \mathrm{mmol}, \mathrm{H}_{2} \mathrm{O}_{2} 15 \mathrm{mmol}, \\
\text { DCE } 15 \mathrm{~mL}, 60^{\circ} \mathrm{C}, 4 \mathrm{~h}\end{array}$ & 23 & 67 & 15.4 \\
\hline 3 & $\mathrm{H}_{4} \mathrm{SiW}_{12} \mathrm{O}_{40}$ & $\begin{array}{c}5 \text { mmol ketone, catalyst } 0.0025 \\
\text { mmol, } \mathrm{H}_{2} \mathrm{O}_{2} 15 \mathrm{mmol}, \\
\text { DCE } 15 \mathrm{~mL}, 60^{\circ} \mathrm{C}, 4 \mathrm{~h}\end{array}$ & 12 & 84 & 10.8 \\
\hline 4 & COOH@PIL & $\begin{array}{c}5 \text { mmol ketone, catalyst } 0.0025 \\
\text { mmol, } \mathrm{H}_{2} \mathrm{O}_{2} 15 \mathrm{mmol}, \\
\text { DCE } 15 \mathrm{~mL}, 60^{\circ} \mathrm{C}, 4 \mathrm{~h}\end{array}$ & 10.2 & 82 & $8.4^{\mathrm{a}}$ \\
\hline 5 & COOH-PIL@P-POM & $\begin{array}{c}5 \text { mmol ketone, catalyst } 0.0025 \\
\text { mmol, } \mathrm{H}_{2} \mathrm{O}_{2} 15 \mathrm{mmol}, \\
\text { DCE } 15 \mathrm{~mL}, 60^{\circ} \mathrm{C}, 4 \mathrm{~h}\end{array}$ & 83.4 & 93 & $77.6^{a}$ \\
\hline 6 & COOH-PIL@Si-POM & $\begin{array}{c}5 \text { mmol ketone, catalyst } 0.0025 \\
\text { mmol, } \mathrm{H}_{2} \mathrm{O}_{2} 15 \mathrm{mmol}, \\
\text { DCE } 15 \mathrm{~mL}, 60^{\circ} \mathrm{C}, 4 \mathrm{~h}\end{array}$ & 68.5 & 90 & $61.6^{\mathrm{a}}$ \\
\hline 7 & COOH-PIL@P-POM & $\begin{array}{c}5 \text { mmol ketone, catalyst } 0.0025 \\
\text { mmol, } \mathrm{H}_{2} \mathrm{O}_{2} 15 \mathrm{mmol} \text {, } \\
\text { DCE } 15 \mathrm{~mL}, 60^{\circ} \mathrm{C}, 4 \mathrm{~h}\end{array}$ & 78.6 & 92 & $72.3^{\mathrm{b}}$ \\
\hline 8 & COOH-PIL@P-POM & $\begin{array}{c}5 \text { mmol ketone, catalyst } 0.0025 \\
\text { mmol, } \mathrm{H}_{2} \mathrm{O}_{2} 15 \mathrm{mmol}, \\
\text { DCE } 15 \mathrm{~mL}, 60^{\circ} \mathrm{C}, 4 \mathrm{~h}\end{array}$ & 87.3 & 85 & $74.2^{\mathrm{c}}$ \\
\hline
\end{tabular}

These facts demonstrate the existence of synergistic catalytic effect between the POM anion and carboxylic functionalized polymeric cation. During the oxidation, the abundant carboxylic acid group in polymeric cation framework and POM anion could be transferred into active peroxide acid and POM peroxo complex in situ with $\mathrm{H}_{2} \mathrm{O}_{2}$, respectively. They acted as active oxidants oxidize the cyclic ketone into product lactone. Moreover, these better results also associated with the mesopore structure with acidic and amphiphilic activity surface, which distributed on the surface of two ionic self-assemblies. On the one hand, they facilitated the adsorption and transfer of reactants and supplied a microenvironment for the evolution of ketone and $\mathrm{H}_{2} \mathrm{O}_{2}$ molecules into the catalyst pore channels. On the other hand, the formed product lactone could be separated immediately, avoiding the over-oxidation and hydrolysis under acidic surroundings. Under the same COOH-PIL conditions, COOH-PIL@P-POM gave better results than COOH-PIL@Si-POM. The reasons lie in the following facts: (1) The higher negative charge on the surface of $\mathrm{SiW}_{12} \mathrm{O}_{40}{ }^{-4}$ caused the obtained ionic self-assembly processes' higher acidity content, which increased the probability of hydrolysis of lactone. Meanwhile, this feature could enhance the cross-linking between nanoparticles, and as a consequence, the number of pores on the surface of ionic self-assembly decreased, and the pore cavity became smaller. (2) $\mathrm{PW}_{12} \mathrm{O}_{40}{ }^{-3}$ based peroxo POM anion species presented higher oxidative activity than that of $\mathrm{SiW}_{12} \mathrm{O}_{40}{ }^{-4}$. The influence of polymeric organic anion on BV oxidation was also remarkable. With the increasing of $\mathrm{FW}$, the conversion of oxidation significantly improved, since the number of carboxylic acid groups and the count of POM anions increased correspondingly. There was a slight decline in selectivity due to the enhancement of oxidative catalytic activity. Moreover, the crosslinking surface morphology of ionic self-assemblies resulting from the increasing of FW could 
impede oxidation proceeding via the limitation of mass transfer of substrate to the active site on the micropore channel. In order to obtain the best oxidation results, an optimization between the synergistic catalytic effect and morphology control should be achieved. The scope and limitation of cyclic ketone was also investigated, and the results were summarized in Table 2. All the selected substrates reacted smoothly and gave the corresponding lactones with good to excellent yields. Among them, 2-adamantanone gave the best result for its large molecular size and rigid structure, which makes it difficult to hydrolysis and leads to high chemoselectivity of the corresponding lactone. Owing to the higher ring strain and lower steric hindrance, cyclobutanone and cyclopentanone gave higher yields than cyclohexanone. Additionally, no substituent effect was observed for mono substituted cyclohexanone, with methyl at the para-, meta-, and ortho-position found to have a minimal effect on the outcome of the oxidation. A little surprise in this work is the oxidation of cycloheptanone; the corresponding lactone was obtained with a yield of $7 \%$. This value is almost double compared with the literature [6]. To demonstrate the scalability and synthetic utility of this present protocol, a hundred-gram scale oxidation of cyclohexanone $(1 \mathrm{~mol})$ was carried out. Under the standard conditions, $\varepsilon$-caprolactone was obtained in the yield of $74 \%$.

Table 2. Results of BV oxidation of various cyclic ketones with $\mathrm{H}_{2} \mathrm{O}_{2}$ catalyzed by two ionic self-assemblies.

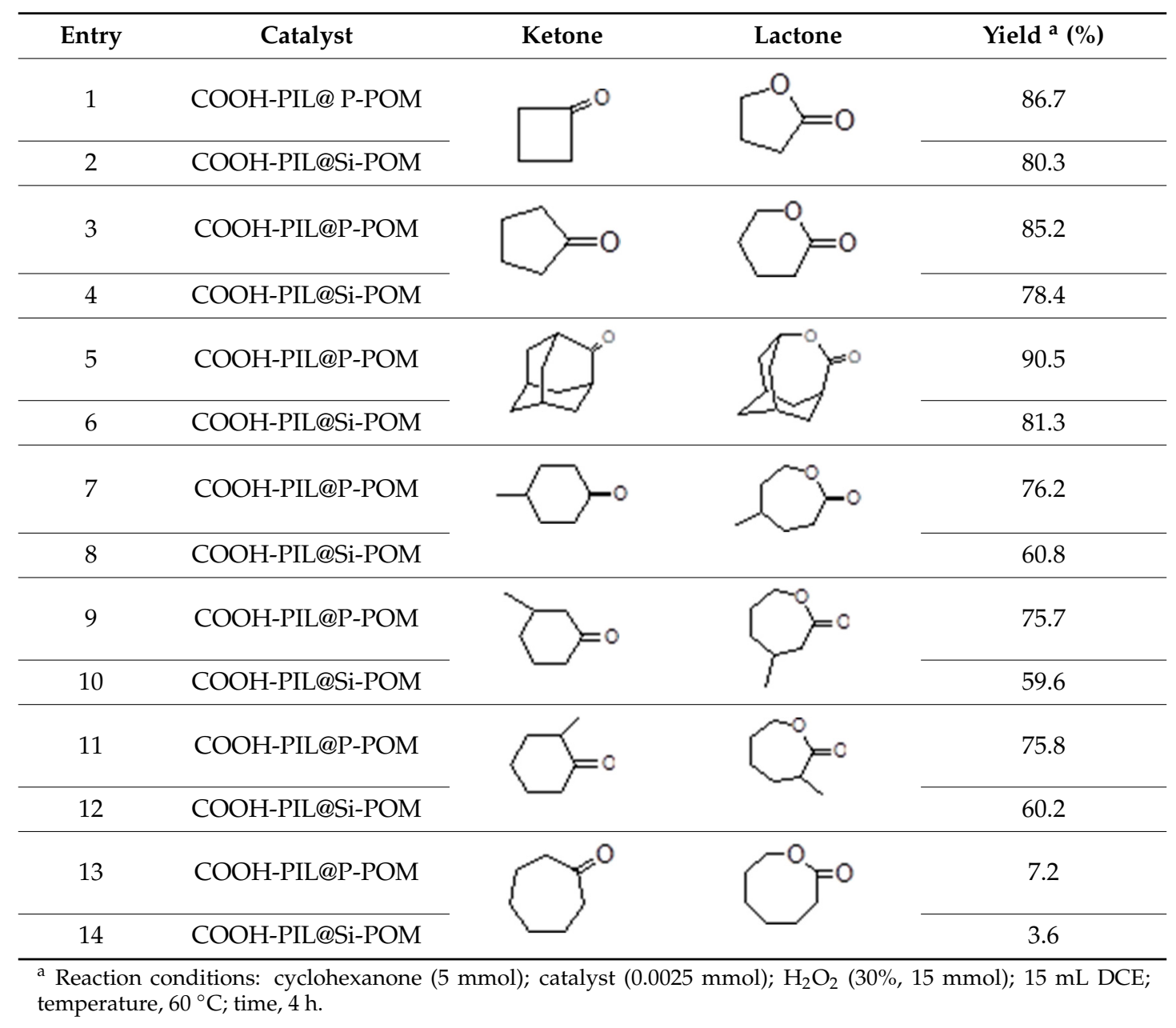

\subsection{Catalyst Reusability}

The reusability of two self-assemblies was then investigated, using cyclopentanone as the model substrate. After the first use, two ionic self-assemblies were separated directly by simple filtration and washed with ethyl ester, dried under vacuum, and subjected to another cycle with a fresh reaction 
mixture. The result was shown in Figure 5. Two self-assemblies could be quantitatively recovered from the reaction mixture and reused consecutively for five times without significant loss in catalytic performance compared with that of the fresh catalyst. Moreover, the TG curve, XRD, IR spectra, and elemental analysis of recycled composites were also determined and found to be very close to the data for fresh catalyst. In addition, ICP analysis of the solution after reaction showed that the POM anion contents were negligible, also indicating that no leaching of active component occurred during the reaction. The high stability and recyclability of this catalytic system offers a potential candidate for an economic and environmentally friendly process for synthesis of lactones in future.

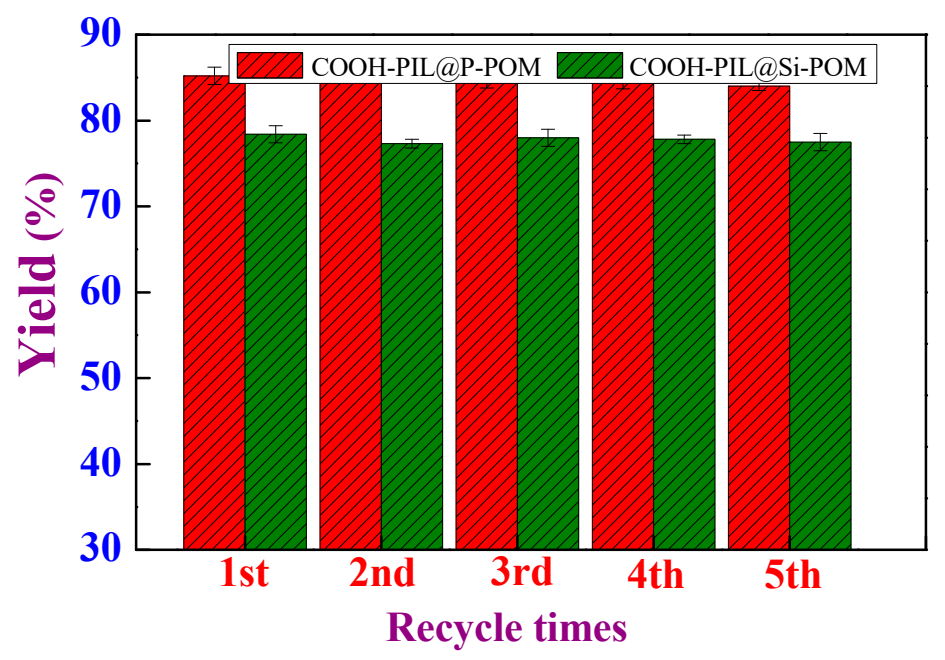

Figure 5. Reusability of COOH-PIL@P-POM and COOH-PIL@Si-POM in BV oxidation of cyclopentanone.

\section{Materials and Methods}

\subsection{Chemicals and Materials}

All chemicals and solvents were commercially available and used as received without further purification. FT-IR spectra were recorded with a Bruker Tensor-27 spectrometer (Ettlinggen, BW, Germany) in the range $400-4000 \mathrm{~cm}^{-1}$. Powder X-ray diffraction (XRD) were studied by a with $\mathrm{Cu}$ anode for scanning ranged from $0^{\circ}$ to $90^{\circ}$ of $2 \theta$ using a Rigaku D/Max 2500 diffractometer (Tokyo, Japan). Scanning electron microscopy (SEM) was studied with a Hitachi S-4800 microscope (Tokyo, Janpan). C, H, and N elemental analyses were performed on a Vario EL III elemental analyzer (Hanau, HD, Germany). The thermal analysis (TGA) was performed using a TGA Q 50 instrument (Newcastle, $\mathrm{DE}, \mathrm{USA}$ ); samples were heated at a constant heating rate of $10^{\circ} \mathrm{C} \cdot \mathrm{min}^{-1}$ room temperature to $400{ }^{\circ} \mathrm{C}$ under $\mathrm{N}_{2}$ atmosphere. Nuclear magnetic resonance (NMR) spectra were recorded on a Bruker AC-400 spectrometer (Ettlinggen, BW, Germany) in appropriate deuterated solvents.

\subsection{Preparation of Ionic Liquid Monomer (COOH-PIL)}

To a mixture of 2-(dimethylamino) ethyl methyacrylate $(4.8 \mathrm{~g}, 30 \mathrm{mmol})$ in $30 \mathrm{~mL}$ acetone, 1-chloro acetic acid $(3.0 \mathrm{~g}, 30 \mathrm{mmol})$ was added in dropwise, and it was stirred at $65{ }^{\circ} \mathrm{C}$ for $24 \mathrm{~h}$ under the protection of nitrogen. On completion, solvent was removed by rotary evaporation. After drying under vacuum, COOH-IL was obtained with a yield of $95 \%$. FT-IR $\left(\mathrm{KBr}, \mathrm{cm}^{-1}\right): 3567,3010,2959,1728$, 1631, 1473, 1390, 1337, 1147. ${ }^{1} \mathrm{H}$ NMR $\left(400 \mathrm{MHz}, \mathrm{D}_{2} \mathrm{O}\right): \delta=6.09(\mathrm{~s}, 1 \mathrm{H}), 5.68(\mathrm{~s}, 1 \mathrm{H}), 4.51(\mathrm{t}, \mathrm{J}=6.8$ $\left.\mathrm{Hz}, 2 \mathrm{H}, \mathrm{O}-\mathrm{CH}_{2}\right), 4.00\left(\mathrm{~m}, \mathrm{~J}=1.5 \mathrm{~Hz}, 2 \mathrm{H},-\mathrm{CH}_{2}\right), 3.65\left(\mathrm{~m}, 2 \mathrm{H},-\mathrm{CH}_{2}\right), 3.21\left(\mathrm{t}, 6 \mathrm{H}, 2-\mathrm{CH}_{3}\right), 1.98(\mathrm{~s}, 3 \mathrm{H}$, $-\mathrm{CH}_{3}$ ) (Figure S1). Anal. Calcd. for $\mathrm{C}_{10} \mathrm{H}_{18} \mathrm{~N}_{1} \mathrm{O}_{4} \mathrm{Cl}: \mathrm{C}, 47.67 ; \mathrm{H}, 7.15 ; \mathrm{N}, 5.15$. Found: C, 47.70; H, 7.24; $\mathrm{N}, 5.11$. 
Polymerization was carried out with AIBA (2,2'-azobis(2-methylpropionamide) dihydrochloride) as the initiator in a degassed tube under $\mathrm{N}_{2}$. In a typical procedure, ionic liquid monomer COOH-IL ( $3 \mathrm{~g}, 16 \mathrm{mmol}), 15 \mathrm{~mL}$ deionized water, and $7 \mathrm{~mL}$ EDTA aqueous solution were charged; the mixture was degassed by bubbling $\mathrm{N}_{2}$ to remove oxygen and was heated to $50^{\circ} \mathrm{C}$. After addition of $15 \mathrm{~mL}$ AIBA aqueous solution $(0.27 \mathrm{~g}, 1.25 \mathrm{mmol})$ in dropwise, the polymerization was carried out at $65^{\circ} \mathrm{C}$ for $12 \mathrm{~h}$ under $\mathrm{N}_{2}$ atmosphere. On completion, the polymers were purified by sedimentation from cold acetone and dried under vacuum at $80^{\circ} \mathrm{C}$ overnight. A total of $2.8 \mathrm{~g} \mathrm{COOH}$-functionalized PILs (COOH-PIL) was obtained in the yield of 91\%. FT-IR( $\left.\mathrm{KBr}, \mathrm{cm}^{-1}\right)$ : 3421, 2962, 1729, 1621, 1474, 1399, $1349,1224,1147$.

${ }^{1} \mathrm{H}$ NMR $\left(400 \mathrm{MHz}, \mathrm{D}_{2} \mathrm{O}\right): \delta=4.02\left(\mathrm{~s}, 2 \mathrm{H},-\mathrm{CH}_{2}\right), 3.62\left(\mathrm{t}, 2 \mathrm{H},-\mathrm{CH}_{2}\right), 2.65\left(\mathrm{t}, 2 \mathrm{H},-\mathrm{CH}_{2}\right), 2.35(\mathrm{~s}$, $\left.6 \mathrm{H}, 2 \mathrm{CH}_{3}\right), 1.78\left(\mathrm{t}, 2 \mathrm{H}, \mathrm{CH}_{2}\right), 0.85\left(\mathrm{~s}, 3 \mathrm{H},-\mathrm{CH}_{3}\right)$ (Figure S1) Anal. Calcd. for $\mathrm{C}_{10 \mathrm{n}} \mathrm{H}_{18 \mathrm{n}} \mathrm{N}_{\mathrm{n}} \mathrm{O}_{4 \mathrm{n}} \mathrm{Cln}: \mathrm{C}$, 47.67; H, 7.15; N, 5.15. Found: C, 47.71; H, 7.19; N, 5.09. The viscosity-average molecular weight was determined by Vickers viscometer (Hangzhou, China), and the values were 15,000, 23,000, and 30,000 , respectively.

\subsection{Preparation of Ionic Self-Assemblies}

In a typical experiment, COOH-PIL ( $0.5 \mathrm{~g}$ ) was thoroughly dissolved in $20 \mathrm{~mL}$ deionized water; then, aqueous solution of $\mathrm{H}_{3} \mathrm{PW}_{12} \mathrm{O}_{40}(1.9 \mathrm{~g}, 0.45 \mathrm{mmol})$ or $\mathrm{H}_{4} \mathrm{SiW}_{12} \mathrm{O}_{40}(1.4 \mathrm{~g}, 0.42 \mathrm{mmol})$ was added in dropwise under vigorous stirring conditions. A precipitate was formed immediately. The suspension was stirred at room temperature for $24 \mathrm{~h}$. Afterwards, the resulting ionic self-assembly was collected by filtration, washed with water, and dried overnight under vacuum at $80^{\circ} \mathrm{C}$ to afford the final products with isolated yields of $89 \%$ and $91 \%$, respectively.

COOH-PL@P-POM, FT-IR (KBr, cm $\left.{ }^{-1}\right)$ : 3408, 2964, 1728, 1620, 1473, 1222, 1145, 1080, 979, 896, 816, 594, 507. Anal. Calcd. for $\mathrm{C}_{10 \mathrm{n}} \mathrm{H}_{18 \mathrm{n}} \mathrm{N}_{\mathrm{n}} \mathrm{O}_{17.33 \mathrm{n}} \mathrm{P}_{0.33 \mathrm{n}} \mathrm{W}_{4 \mathrm{n}}$ : $\mathrm{C}, 10.22 ; \mathrm{H}, 1.54 ; \mathrm{N}, 1.19$. Found: $\mathrm{C}, 10.24 ; \mathrm{H}$, $1.44 ; \mathrm{N}, 1.15$.

COOH-PL@Si-POM, FT-IR (KBr, cm ${ }^{-1}$ ): 3489, 2994, 1727, 1618, 1466, 1232, 1143, 1015, 972, 921, 882, 796, 528. Anal. Calcd. for $\mathrm{C}_{10 \mathrm{n}} \mathrm{H}_{18 \mathrm{n}} \mathrm{N}_{\mathrm{n}} \mathrm{O}_{14 \mathrm{n}} \mathrm{Si}_{0.25 \mathrm{n}} \mathrm{W}_{3 \mathrm{n}}$ : C, 14.79; $\mathrm{H}, 1.94 ; \mathrm{N}, 1.50$. Found: $\mathrm{C}, 14.72$; $\mathrm{H}, 1.88 ; \mathrm{N}, 1.48$.

\subsection{BV Oxidation of Cyclic Ketones}

In a typical experiment, a mixture of cyclic ketone $(10 \mathrm{mmol})$, dichloroethane $(15 \mathrm{~mL})$, COOH-PIL@POM $(0.15 \mathrm{~g})$ were added into a $25 \mathrm{~mL}$ three-necked flask. Under the protection of nitrogen, the reaction mixture was heated up to $70{ }^{\circ} \mathrm{C}$ with vigorous stirring, and aqueous $\mathrm{H}_{2} \mathrm{O}_{2}$ ( $30 \mathrm{wt} \%, 15 \mathrm{mmol}$ ) was added into within $20 \mathrm{~min}$. The reaction was stirred for another $4 \mathrm{~h}$. On completion, the catalyst was separated from the mixture by centrifugation, and the liquid was extracted with ethyl ether $(3 \times 10 \mathrm{~mL})$. Combined the organic layer, it was dried over anhydrous sodium sulfate and collected under vacuum distillation. The product in the resulting mixture was determined and quantitatively analyzed by a gas chromatography-mass spectrometry (GC-MS; PerkinElmer, Clarus SQ-8 equipped with a carpillary column, TC-1701(GL Sciences, length $60 \mathrm{~m}$, i.d. $0.25 \mu \mathrm{m}$, and film thickness $0.25 \mu \mathrm{m}$ ).

The recovered catalyst was washed with ethyl ether, dried under vacuum at $80^{\circ} \mathrm{C}$, and then reused in the next run; the reaction was performed as described above. The concentration of $\mathrm{H}_{2} \mathrm{O}_{2}$ in its aqueous solution was determined iodometrically prior to use in the oxidation reaction. Self-decomposition of $\mathrm{H}_{2} \mathrm{O}_{2}$ as a function of time was monitored by measuring the volume of oxygen evolved.

\section{Conclusions}

In conclusion, we have developed an efficient approach for cyclic ketone additive-free BV oxidation in the presence of $\mathrm{H}_{2} \mathrm{O}_{2}$, using two carboxylic acid functionalized PILs and POM ionic self-assemblies with tunable amphiphilic and oxidative activity and porous structure as heterogeneous catalysts. Under optimized conditions, the target lactones were obtained with isolated yields ranging from 
$59.6 \%$ to $90.5 \%$. There are several noteworthy features of this approach including ionic self-assemblies could be easily prepared using industrial available chemicals, good yield, mild reaction condition, simple workups and purification, high stable reusability of self-assemblies, etc., which make it potentially applicable in industry. This present work suggests that the combination of carboxylic acid functionalized long carbon chain cations with POM anions can work together for the BV oxidation of cyclic ketone. For carboxylic acid functionalized polymeric cation organic frameworks, which not only generated active peracids in situ but also provided a nano-sized micro-environment with amphiphilic activity, which is beneficial for $\mathrm{H}_{2} \mathrm{O}_{2}$ and ketone approaching the catalytic active site on pore cavity, on the other hand, the produced lactones could be removed in time, avoiding the undesirable over-oxidation and hydrolysis under acidic environment. POM anion acted as a rigid pillar node embedded and supported in the 2D frameworks formed by carboxylic functionalized polymeric quaternary ammonium cations, thereby fabricating an open three-dimensional network structure with hierarchical porosity. Meanwhile, POM anion also served as precursor for active catalytic intermediate POM peroxide species.

Supplementary Materials: The following are available online at http://www.mdpi.com/2073-4344/10/1/127/s1, Figure S1: ${ }^{1} \mathrm{H}$ NMP of COOH-IL and COOH-PIL, Figure S2: GC-MS Spectra of Cyclopentanone Oxidation Product, Figure S3: GC-MS Spectra of Cyclohexanone Oxidation Product.

Author Contributions: Conceptualization, investigation, and supervision: X.Z., Q.L., and A.Y.; proposed methodology and supervision: X.L. and H.X.; validation, formal analysis, and writing: Z.X. and H.X. All authors have read and agreed to the published version of the manuscript.

Funding: This research was funded by the National Natural Science Foundation of China (No. 51703090), Research Project of Fujian Province Education Department (No. JAT160373), the Open Project of State Key Laboratory of Structural Chemistry (20170030), and the Research Project of Minjiang University (No. MYK19010).

Acknowledgments: The authors are grateful for the funding from the National Natural Science Foundation of China, Fujian Province Education Department and Minjiang University.

Conflicts of Interest: The authors declare no conflict of interest.

\section{References}

1. Badu, R.P.; Oconnor, K.; Seeram, R. Current progress on bio-based polymers and their future trends. Prog. Biomate. 2013, 2, 8.

2. Zhu, Y.; Romain, C.; Williams, C.K. Sustainable polymers from renewable resources. Nature 2016, 540, 352-362. [CrossRef]

3. Hillmyer, M.A.; Tolman, W.B. Aliphatic polyester block polymers: Renewable, degradable, and sustainable. Acc. Chem. Res. 2014, 47, 2390-2396. [CrossRef]

4. Hillmyer, A.C.; Tolman, W.B. Aliphatic polyesters: Synthesis, properties and applications. Adv. Polym. Sci. 2002, 157, 1-40.

5. Tenbrink, G.J.; Arends, I.W.C.E.; Sheldon, R.A. The-Baeyer-Villiger reaction: New developments toward greener procedures. Chem. Rev. 2004, 104, 4105-4124. [CrossRef] [PubMed]

6. Sitko, M.; Szelwicka, A.; Wojewodka, A.; Skwarek, A.; Tadasiewicz, D.; Schimmelpfennig, L.; Dziuba, K.; Witczakc, M.M.; Chrobok, A. Perdecanoic acid as a safe and stable mediumchain peracid for Baeyer-Villiger oxidation of cyclic ketones to lactones. RSC Adv. 2019, 9, 30012-30018. [CrossRef]

7. Corma, A.; Navarro, M.T.; Renz, M. Lewis acidic Sn (IV) centers-grafted onto MCM-41 as catalytic sites for the Baeyer-Villiger oxidation with hydrogen peroxide. J. Catal. 2003, 219, 242-246. [CrossRef]

8. Chen, T.; Wang, B.; Li, Y.; Liu, L.; Qiu, S. Hydrothermal synthesis of tin contain mesoporous silicas and their catalytic performance over Baeyer-Villiger oxidation of cyclohexanone to $\varepsilon$-carpolactone: Comparison of Sn/MCM-41 and Sn/SBA-15. J. Porrous Mater. 2015, 22, 949-957. [CrossRef]

9. Mandai, K.; Hanata, M.; Mitsudo, K.; Mandai, H.; Hashimoto, H.; Takada, J. Bateriogenic iron oxide as an effective catalyst for Baeyer-Villiger oxidation with molecular oxygen and benzaldehyde. Tetrahedron 2015, 71, 9403-9407. [CrossRef]

10. Llamas, R.; Jimenez-Sanchidrian, C.; Ruiz, J.R. Environmentally friendly Baeyer-Villiger oxidation with $\mathrm{H}_{2} \mathrm{O}_{2} /$ nitrile over $\mathrm{Mg}(\mathrm{OH})_{2}$ and MgO. Appl. Catal. B Environ. 2007, 72, 18-25. [CrossRef] 
11. Thomas, J.M.; Raja, R. Catalytically active centres in porous oxides: Design and performance of highly selective news catalysts. Chem. Commun. 2001, 8, 675-687. [CrossRef]

12. Fischer, J.; Holderich, W.F. Baeyer-Villiger-oxidation of cyclopentanone with aqueous hydrogen by acid heterogeneous catalysis. Appl. Catal. A Gen. 1999, 180, 435-443. [CrossRef]

13. Xing, W.Z.; Ma, Q.G.; Peng, X.H. Silica/A153-SO3H: An efficient catalyst for the Baeyer-Villiger oxidation of cyclic ketones with hydrogen peroxide. C. R. Chim. 2015, 18, 581-585. [CrossRef]

14. Chen, S.Y.; Zhou, X.T.; Wang, J.Z.; Luo, R.C.; Luo, Q.J.; Yu, L.J.; Ji, H.B. Promoting the aerobic Baeyer-Villiger oxidation of ketone over carboxylic multi-walled carbon namotubes. Mol. Catal. 2017, 438, 152-157. [CrossRef]

15. Wei, Y.L.; Yang, Z.W.; Zhao, G.H.; Yang, W. Synthesis and catalytic properties of macroporpus $\mathrm{SiO}_{2}$-coated CNT-sieve-composite supported 12-tungstophosph-oric acid catalysts with dual pore structure for Baeyer-Villiger oxidation of cyclic ketones under microwave irradition. J. Catal. 2019, 371, 196-206. [CrossRef]

16. Yuan, J.; Mecerreyes, D.; Antonietti, M. Poly(ionic liquid)s: An updaye. Prog. Polym. Sci. 2014, $38,1009$. [CrossRef]

17. Leng, Y.; Zhao, J.; Jiang, P.; Wang, J. Amphiphilic polyoxometalate-paired polymer coated Fe3O4: Magnetically recyclable catalyst for epoxidation of bio-derived olefins with $\mathrm{H}_{2} \mathrm{O}_{2}$. ACS Appl. Mater. Interfaces 2014, 6, 5947-5954. [CrossRef]

18. Adhikary, S.D.; Tiwari, A.; Nagaiah, T.C.; Manda, D. Stabilization of cobalt-poly-oxometalate over poly(ionic liquids) compsiites for efficient electrocatalytic water oxidation. ACS Appl. Mater. Interfaces 2018, 10, 38872-38879. [CrossRef]

19. Fafiee, E.; Shahebrahimi, S. Organic-inorganic hybrid polyionic liquid based polyoxomatalate as nano porous material for selective oxidation of sulfides. J. Mole. Struct. 2017, 1139, 255-263.

20. Kim, D.W.; Chi, D.Y. Polymer-supported ionic liquids: Imidazolium salts as catalysts for nucleophilic substitution reaction including fluorinations. Angew. Chem. Int. Ed. 2004, 43, 483-485. [CrossRef]

21. Parvulescu, C.I.; Hardacre, C. Catalysis in ionic liquids. Chem. Rev. 2007, 107, 2615-2665. [CrossRef]

22. Tao, D.J.; Liu, F.J.; Wang, L.; Jiang, L.L. A green and efficient hydration of alkynes catalyzed by hierarchically porous poly(ionic liquid)s. Appl. Catal. A Gen. 2018, 564, 56-63. [CrossRef]

23. Jin, M.J.; Taher, A.; Kang, H.J.; Choi, M.; Ryoo, R. Palladium acetate immobiliz-ed in a hierarchical MFI zeolite-supported ionic liquid: A highly active and recyclable catalyst for Suzuki reaction in water. Green Chem. 2009, 11, 309-313. [CrossRef]

(C) 2020 by the authors. Licensee MDPI, Basel, Switzerland. This article is an open access article distributed under the terms and conditions of the Creative Commons Attribution (CC BY) license (http://creativecommons.org/licenses/by/4.0/). 